\title{
Baixa ingestão de fibras alimentares em idosos: estudo de base populacional ISACAMP 2014/2015
}

\author{
Low intake of dietary fibers among the elderly: \\ 2014/2015 ISACAMP population-based study
}

Graziele Maria da Silva (https://orcid.org/0000-0003-0634-275X) ${ }^{1}$

Daniela de Assumpção (https://orcid.org/0000-0003-1813-996X) ${ }^{2}$

Marilisa Berti de Azevedo Barros (https://orcid.org/0000-0003-3974-195X) ${ }^{2}$

Antônio de Azevedo Barros Filho (https://orcid.org/0000-0001-6239-1121) ${ }^{3}$

Ligiana Pires Corona (https://orcid.org/0000-0001-5298-7714) ${ }^{1}$

\footnotetext{
${ }^{1}$ Laboratório de

Epidemiologia Nutricional,

Faculdade de Ciências

Aplicadas, Universidade

Estadual de Campinas

(UNICAMP). R. Pedro

Zaccaria 1300, Jd. Santa

Luiza. 13484-350

Limeira SP Brasil. gramsilva9@gmail.com

${ }^{2}$ Departamento de Saúde

Coletiva, Faculdade

de Ciências Médicas,

UNICAMP. Campinas SP

Brasil.

${ }^{3}$ Departamento de Pediatria,

Faculdade de Ciências

Médicas, UNICAMP.

Campinas SP Brasil.
}

Resumo O objetivo deste artigo é avaliar a prevalência de inadequação do consumo de fibras alimentares e seus fatores associados em idosos. Estudo transversal de base populacional que avaliou participantes do Inquérito de Saúde do Municipio de Campinas (ISACAMP) em 2014/2015, com 1.074 idosos ( $\geq 60$ anos). O consumo de fibras na dieta foi obtido utilizando um recordatório de 24h, e foi estimada a prevalência de inadequação de fibras de acordo com o ponto de corte do Institute of Medicine (30 g/dia para homens e $21 \mathrm{~g} /$ dia para mulheres). Os fatores avaliados foram condições sociodemográficas (sexo, idade, escolaridade, renda, estado conjugal), de saúde (número de doenças referidas) e estilo de vida (atividade física, tabagismo e etilismo). A prevalência de inadequação do consumo de fibras alimentares foi de $86,6 \%$, sendo maior em homens $(R P=1,10)$, em solteiros ou divorciados $(R P=1,09)$ e inativos fisicamente $(R P=1,07)$. Idosos de maior renda $e$ que relataram ter de 1 a 2 doenças crônicas não transmissíveis tiveram maior ingestão de fibras. A alta prevalência da inadequação do consumo de fibras alimentares em idosos aponta para que sejam desenvolvidas ações de saúde e nutrição a fim de garantir um consumo alimentar adequado destes compostos.

Palavras-chave Idoso, Fibras na dieta, Saúde Pública, Nutrição do Idoso 


\section{Introdução}

O envelhecimento populacional vem aumentando rapidamente a nível mundial ${ }^{1,2}$. Os processos que giram em torno do envelhecimento se associam a diversas alterações fisiológicas e patológicas, como mudanças sensoriais, neuroendócrinas, físicas, bioquímicas e de ordem psicossocial $^{3,4}$.

Um dos principais fatores que pode levar ao inadequado consumo alimentar é a anorexia do envelhecimento, caracterizada pela inapetência e baixa ingestão alimentar, que pode ocorrer devido à diminuição da demanda energética, frequentemente causada pela redução da atividade física, menor palatabilidade dos alimentos, dificuldades de mastigação e deglutição, fumo, etilismo, fatores sociais, clínicos e medicamentosos ${ }^{5,6}$.

As fibras são carboidratos não digeríveis, presentes em alimentos como tubérculos e raízes, cereais integrais, leguminosas, frutas, legumes e verduras ${ }^{7}$. A recomendação do consumo ideal de fibras totais segundo a Dietary Reference Intakes (DRIs) do Institute of Medicine corresponde a aproximadamente $30 \mathrm{~g} /$ dia para homens e 21 $\mathrm{g} /$ dia para mulheres $>50$ anos, em uma dieta de $2.000 \mathrm{kcal}^{8}$.

O consumo adequado desses compostos produz efeitos fisiológicos benéficos no organismo, como na redução da pressão arterial, melhora nos níveis de lipídios séricos e redução dos indicadores inflamatórios ${ }^{910}$. Também atuam na prevenção e no controle de doenças cardiovasculares, diabetes mellitus, obesidade, e na melhora da motilidade intestinal ${ }^{11-15}$.

As fibras também desempenham uma função prebiótica, um exemplo é a função do butirato, produto da fermentação em forma de ácidos graxos de cadeia curta (AGCC), e que atua como imunomodulador intestinal ${ }^{16}$. Além disso, estudos evidenciam a atuação das fibras na melhora da absorção de cálcio, na estimulação dos hormônios atuantes na regulação da densidade mineral óssea ${ }^{17}$ e no alongamento de telômeros correspondendo a um menor envelhecimento biológico $^{18}$.

Mesmo estes benefícios sendo amplamente conhecidos e divulgados, a inadequação do consumo desse composto alimentar é prevalente em diversos países ${ }^{19,20}$ inclusive no Brasil ${ }^{21}$. Um estudo desenvolvido com 1.510 idosos mostrou $90,1 \%$ de inadequação do consumo de fibras alimentares $^{22}$. Essa elevada inadequação vem sendo explicada pela transição nutricional pela qual o país está passando atualmente. A Pesquisa de Or- çamento Familiar (POF), que avaliou a evolução na disponibilidade de alimentos nas áreas metropolitanas do Brasil entre 1974 e 2009, mostrou um declínio no consumo de alimentos base (arroz e feijão) e de alimentos ricos em fibras alimentares, ao mesmo tempo em que houve um aumento significativo no consumo de produtos refinados ou ultraprocessados, além do consumo excessivo de açúcar e a alta ingestão de gorduras ${ }^{23,24}$.

O consumo de alimentos ricos em fibras pode estar prejudicado por fatores sociais, considerando o maior valor monetário dos vegetais in natura e cereais integrais. Dados da Pesquisa Nacional de Saúde (PNS) de 2013 mostraram que apenas $40,1 \%$ dos idosos (60 anos ou mais) relataram o consumo regular de frutas e hortaliças ( $\geq 5$ vezes ao dia $)^{25}$. Dados da POF 2008-2009 também mostram que o consumo de alimentos ricos em fibras alimentares como as frutas e vegetais, é mais elevado na população com maior renda ${ }^{7}$.

Nesse contexto, considerando que os estudos sobre o consumo de fibras alimentares entre os idosos são escassos e que esta parcela da população é susceptível a apresentar um consumo dietético inadequado, o objetivo do presente estudo foi avaliar o consumo médio de fibras alimentares, a prevalência de inadequação em relação à recomendação vigente, e seus fatores associados em idosos participantes do Inquérito de Saúde do município de Campinas (ISACAMP 2014/2015).

\section{Métodos}

\section{Delineamento do estudo}

Este é um estudo transversal, de base populacional, no qual foram avaliados os dados de 1.074 indivíduos ( $>60$ anos), participantes do Inquérito de Saúde do Município de Campinas (ISACAMP), e do ISACAMP Nutri, realizado no município de Campinas entre os anos de 2014 e 2015. Considerando que este é um estudo populacional com objetivo de abranger amostras representativas das populações adolescente, adulta e idosa do município, a estimativa amostral levou em consideração as distribuições absoluta e relativa da população de Campinas segundo esses domínios. Optou-se pelo sorteio de amostras de pelo menos 1.000 pessoas, para adolescentes e idosos, e de 1.400 para adultos. A amostra foi estratificada por conglomerados em dois estágios, setor censitário e domicílio. Os setores foram sorteados com probabilidade proporcional ao tamanho, dado pelo número de domicílios parti- 
culares permanentes contados no Censo de 2010. Os domicílios foram selecionados por sorteio sistemático, a partir da relação de endereços obtidos em trabalho de campo. Mais informações sobre a amostragem estão disponíveis na página do estudo na internet ${ }^{26}$. No presente trabalho foram analisados somente os idosos.

A coleta de dados foi dividida em dois momentos. Na primeira visita pré-agendada, entrevistadores devidamente treinados questionavam os indivíduos sobre os aspectos socioeconômicos, de saúde e estilo de vida referentes ao questionário do ISACAMP. Na impossibilidade de o idoso responder, quando possível o questionamento era direcionado ao responsável ou cuidador.

A segunda visita era agendada para a realização das perguntas contidas no ISACAMP Nutri, que foi um projeto acoplado ao primeiro (ISACAMP), e foram coletadas informações de consumo alimentar e as avaliações nutricionais. Entre estas informações, procedeu-se a aplicação de um recordatório de 24 horas (REC24H), que consiste no levantamento e quantificação dos alimentos e bebidas consumidos referentes às 24 horas anteriores à entrevista ${ }^{27}$. O REC24H foi conduzido por nutricionistas treinados, utilizando o Multiple-Pass Method (MPM) - técnica proposta pelo Departamento de Agricultura dos Estados Unidos, que visa garantir a qualidade das informações coletadas utilizando uma sistematização da entrevista em passos ${ }^{28}$. Além disso, as entrevistas foram realizadas em diferentes dias da semana e meses do ano para conseguir captar as variedades do consumo. Foram utilizados materiais ilustrativos para inquéritos alimentares para facilitar a identificação das porções dos alimentos $^{29}$.

O projeto ISACAMP 2014/2015 foi aprovado pelo Comitê de ética da FCM/UNICAMP. Todos os procedimentos de pesquisa foram realizados somente após a concordância do participante com o estudo e a assinatura do Termo de Consentimento Livre e Esclarecido (TCLE).

\section{Variáveis e categorias}

Para a variável dependente foi estimada a média de ingestão de fibras totais gramas/dia com base no recordatório de 24 horas segundo as principais fontes alimentares (tubérculos e raízes, cereais integrais, leguminosas, frutas, verduras e legumes). $\mathrm{O}$ valor nutricional dos alimentos foi estimado com base no software Nutrition Data System for Research, (NCC Food and Nutrient Data base, Universidade de Minnesota, EUA).
O programa contém mais de 18.000 alimentos e 7.000 marcas de produtos, também é possível incluir receitas de preparações que não constam no banco de dados do sistema, permitindo que alimentos regionais brasileiros possam ser avaliados e contabilizados de acordo com suas receitas. $\mathrm{Na}$ análise de consistência dos dados de energia, utilizou-se mínimo de 800 kcal e máximo de $3500 \mathrm{kcal}$.

Através do consumo médio de fibras foi estipulada a prevalência de inadequação de acordo com as recomendações propostas pelo Institute of Medicine-US, Dietary Reference Intakes (DRIs) sendo de $30 \mathrm{~g} /$ dia para homens e $21 \mathrm{~g} /$ dia para mulheres acima dos 50 anos em uma dieta de $2.000 \mathrm{kcal}^{8}$.

Para avaliação dos fatores associados ao consumo das fibras alimentares, foram consideradas como variáveis independentes aqueles presentes no protocolo que poderiam afetar o consumo alimentar de modo amplo, com base na literatura disponível. Elas foram divididas em dois blocos hierarquizados, sendo consideradas variáveis distais (bloco 1) as condições sociais, que são associadas tanto diretamente ao consumo alimentar, quanto indiretamente às condições de saúde e estilo de vida ${ }^{30-32}$ e variáveis proximais (bloco 2 ) aquelas referentes ao estilo de vida e saúde, que afetam diretamente o consumo alimentar ${ }^{4,25,31,33}$.

Desta forma, as características incluídas em cada bloco foram:

- Bloco 1 - Condições socioeconômicas: sexo (masculino e feminino); faixa etária (de 60 a 69 anos, 70 a 79 anos e $\geq 80$ anos), escolaridade (categorizada em analfabetos, ensino até a $4^{\circ}$ série e ensino fundamental ou superior), estado conjugal (sendo casados ou união estável, viúvos e solteiros ou divorciados), e renda familiar per capita em salários mínimos com o valor vigente em 2014 de $\mathrm{R} \$ 724,00^{34}$, classificada em $<1$ salários; entre 1 a 2 salários e $>2$ salários.

- Bloco 2 - Condições de saúde e estilo de vida: Em relação as condições de saúde foram o número de doenças crônicas autorrelatadas (hipertensão, diabetes, doença do coração, reumatismo/artrite/artrose, osteoporose e problemas de circulação categorizada em: de 1 a 2 doenças e 3 ou mais doenças). Para o estilo de vida foram incluídas a prática de atividade física, avaliada pelo IPAQ (Questionário Internacional de Atividade Física $)^{35}$ onde foram categorizadas em: ativos, insuficientemente ativos e inativos; o habito de fumar categorizados em (nunca fumou e fumante ou ex-fumante) e o consumo de álcool (categorizados em não etilista e ex-etilista ou etilista). 


\section{Análise dos dados}

Para a análise dos dados, foram estimadas as médias do consumo e seus respectivos erros-padrão para as variáveis contínuas e proporções para as variáveis categóricas. Para a estimativa da diferença dos grupos foram realizados testes de igualdade das médias de Wald e teste qui-quadrado com correção de Rao-Scott, que consideram pesos amostrais com estimativas populacionais.

Para a análise dos fatores associados à inadequação do consumo de fibras totais, realizou-se a regressão de Poisson para calcular os valores razões de prevalência (RP) brutas em uma análise bivariada utilizando como variável dependente o consumo inadequado de fibras alimentares. Para o modelo ajustado, foi estabelecido um modelo hierárquico com inclusão dessas variáveis independentes nos dois diferentes blocos: distal (condições socioeconômicas) e o proximal (condições de saúde e estilo de vida). Aquelas variáveis que apresentaram $o$ valor de $p<0,20$ na análise bivariada foram testadas na análise ajustada. Permaneceram no modelo final aquelas variáveis que apresentaram o valor de $\mathrm{p}<0,05$ ou ajustaram as estimativas anteriores. Todas as análises foram realizadas utilizando o software Stata versão $14^{\circledR}$, em módulo de survey, ou seja, adotando os pesos amostrais atribuídos à amostra complexa, garantindo a representatividade da população.

\section{Resultados}

A amostra de 1.074 idosos foi caracterizada em sua maioria por mulheres $(60 \%, \mathrm{n}=648)$, com idades predominantes entre 60 e 69 anos (50,8\%, $\mathrm{n}=546)$, eram casados ou moravam juntos ( $54 \%$, $\mathrm{n}=573$ ), possuíam a renda salarial per capita superior a 2 salários mínimos $(36 \%, \mathrm{n}=382)$, relataram possuir mais de 3 doenças crônicas não transmissíveis $(74 \%, \mathrm{n}=779)$ e que eram inativos fisicamente $(70 \%, \mathrm{n}=750)$.

O consumo de fibras alimentares pela população foi inferior às recomendações dietéticas, sendo a média de 14,6g/dia com $86,6 \%$ de prevalência de inadequação. Na Tabela 1, são apresentados os valores médios do consumo de fibras alimentares (totais), a prevalência de inadequação (\%) e os valores da razão de prevalência bruta (RP) da regressão bivariada, segundo características socioeconômicas, condições de saúde e estilo de vida.

O consumo médio de fibras totais em relação ao sexo foi maior entre os homens em relação às mulheres, porém quando estratificados segundo as recomendações nutricionais para os diferentes sexos, os homens apresentaram maior prevalência de inadequação. Esse consumo inadequado também foi mais frequente entre idosos de menor idade, analfabetos, com menor renda, inativos fisicamente e nos fumantes ou ex-fumantes (Tabela 1).

Para a análise de regressão de Poisson ajustada aos fatores associados à inadequação do consumo, no modelo 1 (bloco distal), as características que se mostraram associadas foram ser do sexo masculino, solteiro ou divorciado e possuir os menores salários. No modelo final ajustado com a inclusão do bloco proximal (modelo 2) a variável de menor renda deixou de ser significativa e as variáveis de multimorbidade (possuir de 1 a 2 doenças crônicas) e inatividade física não se mostraram associadas, permanecendo significantes somente as variáveis de sexo e estado conjugal (Tabela 2).

Além disso, a inatividade física não mostrou associação com o consumo inadequado de fibras no modelo ajustado, porém a renda teve uma associação inversa com o nível de atividade física $(\mathrm{p}<0,001)$ : quanto menor o salário do indivíduo maior é a prevalência de inatividade física (dados não apresentados).

\section{Discussão}

O presente estudo de base populacional mostrou uma elevada inadequação do consumo de fibras alimentares em idosos, cerca de $86 \%$, com consumo médio de $14,6 \mathrm{~g} / \mathrm{dia}$, inferior às recomendações nutricionais propostas para essa população. Os grupos mais vulneráveis foram do sexo masculino, solteiros ou divorciados, de menor renda, analfabetos e inativos fisicamente.

Os primeiros resultados referentes ao consumo de fibras alimentares em idosos foram publicados anteriormente em estudo transversal como base no Inquérito de Saúde de Campinas (ISACAMP) realizados nos anos de 2008/2009, no qual foram avaliados 1.510 idosos $>60$ anos. $\mathrm{O}$ estudo mostrou uma inadequação do consumo de fibras alimentares em $90,1 \%$ com a média ingestão de $13,5 \mathrm{~g} /$ dia pela população $0^{22}$. Ao comparar com os resultados do presente estudo (média ingestão de 14,6 g e 86,6\% de inadequação), podemos observar um aumento discreto do consumo de fibras.

É possível levantar a hipótese que esse aumento pontual possa ser explicado pela melhoria do acesso à informação, levando a uma maior 
Tabela 1. Consumo de fibras alimentares totais em gramas (FAg), segundo características socioeconômicas e estilo de vida.

\begin{tabular}{|c|c|c|c|c|}
\hline Variáveis e categorias & $\mathbf{n}$ & FAg (IC95\%) & Inadequação (\%) & RP (bruto e IC95\%) \\
\hline Total & 1.074 & $14,66(13,75 ; 15,58)$ & 86,6 & - \\
\hline \multicolumn{5}{|l|}{ Sexo } \\
\hline Feminino & 648 & $13,92(12,61 ; 15,24)$ & 83,7 & 1,00 \\
\hline Masculino & 426 & $15,63(14,68 ; 16,58)^{\star}$ & 91,6 & $1,09(1,03 ; 1,16)^{\star}$ \\
\hline \multicolumn{5}{|l|}{ Idade (em anos) } \\
\hline $60-69$ & 546 & $14,75(13,71 ; 15,78)$ & 87,5 & 1,00 \\
\hline $70-79$ & 336 & $14,76(13,34 ; 16,18)$ & 86,1 & $0,98(0,91 ; 1,05)$ \\
\hline 80 e mais & 189 & $14,19(11,68 ; 16,71)$ & 85,1 & $0,97(0,88 ; 1,07)$ \\
\hline \multicolumn{5}{|l|}{ Estado conjugal } \\
\hline Casados/Amasiados & 573 & $15,35(14,05 ; 16,66)$ & 87,2 & 1,00 \\
\hline Viúvos & 348 & $13,96(12,41 ; 15,50)$ & 83,0 & $0,97(0,89 ; 1,05)$ \\
\hline Solteiros/Divorciados & 153 & $13,43(12,36 ; 14,50)^{\star}$ & 92,8 & $1,06(1,00 ; 1,14)$ \\
\hline \multicolumn{5}{|l|}{ Escolaridade } \\
\hline Fundamental/superior & 411 & $15,61(14,39 ; 16,84)$ & 86,6 & 1,00 \\
\hline Primário ( $4^{\circ}$ ano $)$ & 522 & $14,47(13,29 ; 15,65)$ & 86,0 & $1,00(0,95 ; 1,06)$ \\
\hline Analfabetos & 141 & $12,23(10,32 ; 14,15)^{\star}$ & 89,4 & $1,05(0,98 ; 1,13)$ \\
\hline \multicolumn{5}{|c|}{$\begin{array}{l}\text { Renda familiar per capta } \\
\text { (salário mínimo) }\end{array}$} \\
\hline$<1$ & 324 & $13,60(12,09 ; 15,11)$ & 90,2 & 1,00 \\
\hline $1-2$ & 367 & $13,62(12,51 ; 14,73)$ & 90,1 & $1,00(0,95 ; 1,05)$ \\
\hline$>2$ & 382 & $16,43(15,11 ; 17,75)^{\star}$ & 82,7 & $0,92(0,84 ; 1,00)^{\star}$ \\
\hline \multicolumn{5}{|c|}{$\begin{array}{l}\text { Número de equipamentos na } \\
\text { residência }\end{array}$} \\
\hline 1 a 5 & 183 & $13,48(12,09 ; 14,87)$ & 87,4 & 1,00 \\
\hline 6 a 10 & 454 & $14,74(13,24 ; 16,23)$ & 85,7 & $0,98(0,92 ; 1,05)$ \\
\hline$>11$ & 436 & $15,04(13,65 ; 16,42)$ & 87,4 & $0,99(0,92 ; 1,07)$ \\
\hline \multicolumn{5}{|l|}{ Atividade física } \\
\hline Ativo & 309 & $16,08(14,64 ; 17,51)$ & 82,8 & 1,00 \\
\hline Insuficiente ativo & 103 & $17,34(13,64 ; 21,06)$ & 76,7 & $0,93(0,81 ; 1,06)$ \\
\hline Inativo & 662 & $13,48(12,60 ; 14,36)^{\star}$ & 90,0 & $1,07(1,00 ; 1,15)^{\star}$ \\
\hline \multicolumn{5}{|l|}{ Tabagismo } \\
\hline Não Fumantes & 739 & $15,00(13,96 ; 16,04)$ & 85,1 & 1,00 \\
\hline Fumantes & 335 & $14,00(12,76 ; 15,24)$ & 90,1 & $1,05(0,97 ; 1,14)$ \\
\hline \multicolumn{5}{|l|}{ Consumo de álcool } \\
\hline Não etilista & 852 & $14,43(13,51 ; 15,38)$ & 87,2 & 1,00 \\
\hline Etilista & 222 & $15,51(13,83 ; 17,20)$ & 87,0 & $1,00(0,93 ; 1,07)$ \\
\hline \multicolumn{5}{|l|}{ Número de doenças crônicas } \\
\hline 1-2 doenças & 569 & $14,48(13,40 ; 15,56)$ & 88,7 & 1,00 \\
\hline$>3$ doenças & 486 & $15,00(13,74 ; 16,27)$ & 83,9 & $0,94(0,88 ; 1,00)^{*}$ \\
\hline
\end{tabular}

Nota: ${ }^{*} \mathrm{p}<0,05$ na comparação das categorias da variável em relação à categoria de referência. $\mathrm{RP}=$ razão de prevalência.

Fonte: Dietary Reference Intakes (DRIs) do Institute of Medicine, 2009.

preocupação com a saúde, e consequentemente obtém-se uma melhora nos hábitos alimentares, através de refeições mais saudáveis e a maior independência em idosos ${ }^{36,37}$. Um estudo de coorte realizado em Florianópolis-SC, com 1.790 idosos de 60 anos e mais, encontrou que a autopercep- ção positiva de saúde aumentou $15,2 \%$ e $44 \%$ dos idosos mantiveram essa percepção, fato esse que pode estar relacionado a sua maior independência e autonomia ${ }^{38}$. É possível esperar que o maior acesso à informação poderia impactar de forma positiva na adoção de hábitos mais saudá- 
Tabela 2. Fatores associados a inadequação do consumo de fibras alimentares nos modelos de regressão múltipla de Poisson.

\begin{tabular}{|c|c|c|c|c|}
\hline \multirow{2}{*}{ Variáveis e categorias } & \multicolumn{2}{|c|}{ Modelo $1^{\text {a }}$} & \multicolumn{2}{|c|}{ Modelo $2^{\text {b }}$} \\
\hline & $\mathbf{R P}$ & IC95\% & RP & IC95\% \\
\hline Sexo masculino & 1,10 & $(1,03 ; 1,17)^{\star}$ & 1,11 & $(1,03 ; 1,18)^{\star}$ \\
\hline Idade (anos) & 1,00 & $(0,99 ; 1,00)$ & 1,00 & $(0,99 ; 1,00)$ \\
\hline Estado conjugal & - & - & - & - \\
\hline Casados/Amasiados & 1,00 & - & 1,00 & - \\
\hline Viúvos & 1,00 & $(0,92 ; 1,08)$ & 1,02 & $(0,94 ; 1,10)$ \\
\hline Solteiros/Divorciados & 1,08 & $(1,02 ; 1,15)^{\star}$ & 1,09 & $(1,02 ; 1,17)^{\star}$ \\
\hline Renda familiar per capta (salários mínimos) & - & - & - & - \\
\hline$<1$ & 1,00 & - & 1,00 & - \\
\hline $1-2$ & 0,98 & $(0,93 ; 1,04)$ & 0,99 & $(0,94 ; 1,04)$ \\
\hline$>2$ & 0,91 & $(0,84 ; 0,98)^{\star}$ & 0,94 & $(0,87 ; 1,00)$ \\
\hline Número de doenças crônicas & - & - & - & - \\
\hline $1-2$ & - & - & 1,00 & - \\
\hline$>3$ & - & - & 0,96 & $(0,90 ; 1,03)$ \\
\hline Atividade Física & - & - & - & - \\
\hline Ativo & - & - & 1,00 & - \\
\hline Insuficientemente ativo & - & - & 0,93 & $(0,83 ; 1,06)$ \\
\hline Inativo & - & - & 1,07 & $(1,00 ; 1,15)$ \\
\hline
\end{tabular}

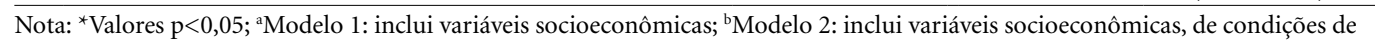
saúde e indicadores de estilo de vida. $\mathrm{RP}=$ razão de prevalência.

Fonte: Dietary Reference Intakes (DRIs) do Institute of Medicine, 2009.

veis, consequentemente o aumento do consumo de alimentos fontes de fibras alimentares, porém, o consumo ainda se encontra distante do que seria o ideal segundo as recomendações ${ }^{8}$.

O resultado aqui apresentado se assemelha a resultados anteriores, provenientes de populações de outros países, como dos Estados Unidos. Um estudo que avaliou o consumo de fibras alimentares na população americana utilizando a base de dados do NHANES, (National Health and Nutrition Examination Survey), avaliou o consumo médio desse nutriente entre os anos de 1999 a 2008, mostrou que o consumo de fibras alimentares na população sendo adultos e idosos se manteve entre $15,6 \mathrm{~g} / \mathrm{dia}$ a $15,9 \mathrm{~g} / \mathrm{dia}^{39}$. Outro estudo que utilizou o mesmo banco de dados do ano 2000 , apontou que $84 \%$ da população americana possuí a ingestão de fibras alimentares inadequada ${ }^{40}$. A partir desses dados, os órgãos de saúde daquele país passaram a aumentar o incentivo do consumo desse nutriente através de campanhas de divulgação pela mídia e incentivaram a realização de mais estudos para essa finalidade ${ }^{39}$.

Em estudo com a base de dados NHANES dos anos de 2011-2014, realizada em idosos >51 anos, mostrou que os alimentos contendo grãos integrais, forneceram uma porcentagem maior de concentração de outros nutrientes como tiamina e niacina além das fibras alimentares, quando comparados com outros alimentos ${ }^{41}$, evidenciando assim que as fontes de fibras alimentares abrangem uma gama de outros nutrientes fundamentais para o bom funcionamento do organismo. Tal estratégia de divulgação poderia ser adotada como uma alternativa para aumentar o consumo desse nutriente pela população brasileira.

Em relação ao consumo de fibras segundo sexo, os resultados aqui apresentados mostraram que os homens tiveram o maior consumo médio em relação às mulheres, porém a prevalência de inadequação foi maior entre os homens. Isso se explica devido às recomendações de fibras alimentares serem maiores para o sexo masculino (30 g/dia, e $21 \mathrm{~g} /$ dia para o sexo feminino). Em outros estudos que avaliaram a ingestão de fibras alimentares, essa prevalência mais elevada de inadequação para o sexo masculino também foi observada ${ }^{22,42}$. Além disso, é sabido que a percepção de saúde pelos homens é menor do que as mulheres, já que tendem a procurar menos o serviço de saúde quando comparados com o sexo feminino ${ }^{43}$. Em um estudo que avaliou as 
condições de saúde e o uso de serviços de saúde da população idosa brasileira, com 340 mil indivíduos ( $\geq 60$ anos) incluídos na amostra da Pesquisa Nacional por Amostra de Domicílios (PNAD), 1998, 2003 e 2008, as mulheres foram o público que obteve o predomínio na busca desses serviços ao longo desses anos ${ }^{44}$. Essa preocupação com a saúde pode levar as mulheres a obterem padrões alimentares qualitativamente melhores, com um maior consumo de alimentos ricos em nutrientes e fontes de fibras alimentares como vistos em outros estudos ${ }^{45,46}$.

Os achados do presente estudo sobre o estado civil mostraram que idosos que possuíam as maiores chances de apresentarem inadequação do consumo de fibras eram viúvos, solteiros ou divorciados quando comparados com indivíduos casados ou em união estável, o que corrobora outros dados encontrados na literatura, em que idosos que tinham cônjuge possuíam a melhor qualidade da dieta com alimentos ricos em fibras alimentares. Em estudo de coorte retrospectivo, que avaliou 86.419 indivíduos, e analisou 4 índices de qualidade da dieta diferentes como o Healthy Eating Index-2010 (HEI-2010), o Alternative Healthy Eating Index-2010 (AHEI-2010), o Alternate Mediterranean Diet (aMED), e a Dietary Approaches to Stop Hypertension (DASH), encontrou que tanto os homens quantos as mulheres acima de 50 anos, que possuíam a melhor qualidade na dieta eram indivíduos casados, de maior renda, ativos fisicamente e possuíam o maior grau de escolaridade ${ }^{47}$.

Em relação às condições socioeconômicas, a prevalência de inadequação foi maior para aqueles que possuíam menor renda. Esse resultado também já foi descrito em outros estudos, onde o menor nível de escolaridade e as menores rendas se mostraram associados com o menor consumo de alimentos fontes de fibras alimentares. Em estudo transversal de base populacional de 416 idosos usuários das unidades básicas de saúde/ SUS e residentes em Goiânia-GO em 2008/2009, que avaliou o consumo diário de frutas, verduras e legumes nessa população, encontrou que o consumo desses alimentos aumentava significativamente conforme o nível de escolaridade e a renda dos indivíduos ${ }^{48}$. $\mathrm{O}$ autor discute que as investigações realizadas no Brasil mostraram que o gênero, o poder aquisitivo e a escolaridade/informação são determinantes para a alimentação equilibrada nestas populações.

Para os aspectos de estilo de vida, os idosos que referiram ser inativos fisicamente apresentaram prevalência mais elevada de inadequação do consumo de fibras alimentares, e esse fato poderia ser explicado pelas diferenças em relação a estilo de vida, em que indivíduos que se preocupam mais com a sua saúde praticam mais atividade física e mantém hábitos alimentares mais saudáveis como observado em outros estudos ${ }^{45,46}$. No entanto, essa associação não se manteve após o ajuste do modelo final. Isso pode em parte ser explicado porque, no presente estudo, a inatividade física teve uma associação inversa importante com a renda, ou seja, naqueles que possuíam os menores salários a prevalência de inatividade física se manteve maior. Esse fato mostra que a renda continua sendo um fator determinante para adoção de um estilo de vida mais saudável através da prática de exercícios físicos, como visto em outros estudos ${ }^{49}$.

Em relação às doenças crônicas, observa-se que aqueles idosos que referiram possuir de uma a duas doenças crônicas se mostram com a maior porcentagem de inadequação do consumo de fibra alimentar, o que pode parecer um resultado contraditório. No entanto, por tratar-se de um estudo transversal, é importante lembrar que os idosos que referiram doenças podem já ter passado por orientação nutricional e mudança de estilo de vida decorrentes do diagnóstico. Os efeitos positivos da fibra alimentar em relação à melhora e ou controle dessas doenças já são bastante conhecidos na literatura, indivíduos com maior aporte de fibras alimentares em sua alimentação obtiveram melhora do seu quadro de saúde como redução do sobrepeso e melhora na composição corporal $^{50}$, diabetes, hipertensão, doenças cardiovasculares $^{51}$, circulatórias, entre outras.

Algumas limitações do presente estudo devem ser levadas em consideração na interpretação dos seus resultados. Para avaliação do consumo através do recordatório de 24 horas, não é possível avaliar o consumo alimentar habitual dos indivíduos e sim o seu consumo no dia anterior ou atual. Além disso, o método também é sensível à memória dos participantes, que pode estar comprometida em idosos, e também depende da qualidade da entrevista e da técnica envolvida. Contudo, este método é o mais comum utilizado em inquéritos populacionais devido a facilidade de aplicação, baixo custo e maior grau de detalhamento de porções. Ademais, por se tratar de uma amostra representativa da população de uma grande cidade, o consumo médio tende a ser similar ao consumo habitual, eliminando os valores extremos devido à distribuição populacional. Também é aplicado em idosos pelo fato de apresentarem uma alimentação monótona na 
maioria das vezes, variando pouco entre os dias $^{52}$. Por fim, para minimizar os possíveis vieses na aplicação do método, os entrevistadores foram treinados utilizando a técnica MPM, conforme descrito nos métodos, visando obter o maior detalhamento possível.

Outra limitação se refere à característica transversal do estudo, não sendo então possível avaliar se as associações aqui apresentadas teriam relação de causa e efeito. No entanto, os inquéritos populacionais são importantes para levantar possíveis fatores associados, gerar hipóteses, e direcionar adequadamente futuras pesquisas longitudinais ou de intervenção.

Contudo, este estudo apresenta pontos fortes. Primeiro, trata-se de uma amostra populacional representativa, com rigor metodológico na amostragem, coleta e manipulação dos dados, motivo pelo qual os achados aqui reportados podem ser extrapolados a nível populacional. Além disso, poucos estudos se referem ao consumo de fibras baseados em média de ingestão ${ }^{33}$, a maioria dos estudos disponíveis analisa suas fontes em frutas, verduras e legumes como aproximação de sua quantidade $\mathrm{f}^{53-55}$. O feijão, por exemplo, que é um alimento rico em fibras, está na base da alimentação do brasileiro, especialmente do idoso, que tende a manter uma alimentação mais próxima do tradicional ${ }^{7}$, ou seja, considerar esses alimentos como fontes importantes de fibras é necessário, e isso só é possível com a quantificação do composto em si e não somente dos grupos alimentares.

Apesar do consumo de alimentos integrais ainda ser baixo na população, ele vem aumentando pela população idosa quando comparados com adolescentes e adultos nos últimos anos ${ }^{56}$. Contudo, ainda é necessário adotar medidas de incentivo ao consumo desse nutriente, assim como a realização de mais estudos, a fim de analisar a ingestão de fibras alimentares em idosos levando em consideração outras variáveis como a influência de medicamentos na alimentação, estado nutricional e outros fatores que podem determinar as escolhas dos alimentos fonte de fibras alimentares.

\section{Conclusão}

O consumo médio de fibras alimentares foi de 14,66 g nos idosos do estudo, valor aquém das recomendações de consumo atuais, com prevalência de inadequação de $86,6 \%$, sendo que os fatores associados à esta inadequação foram sexo masculino e ser solteiro ou divorciado.

Estes achados apontam para que as ações de saúde envolvidas na nutrição do idoso devam ser direcionadas para garantir um consumo alimentar adequado destes compostos, abrangendo vários aspectos envolvidos no acesso à alimentação adequada, desde educação nutricional da população, incentivando o consumo de cereais integrais, além de políticas para melhoria no acesso a alimentos in natura, manutenção da funcionalidade da população idosa e adequado cuidado à saúde. Estudos futuros de intervenção são necessários para que se determine quais seriam as estratégias mais eficazes para melhorar o consumo de fibras nos idosos brasileiros.

\section{Colaboradores}

GM Silva trabalhou na análise de dados e redação do manuscrito. LP Corona trabalhou na concepção do estudo, plano de análises e revisão da redação do manuscrito. D Assumpção trabalhou na coleta de dados, preparação do banco de dados e revisão do manuscrito. MBA Barros e AA Barros Filho trabalharam na coordenação da pesquisa, na revisão da análise e do manuscrito. 


\section{Referências}

1. Saad PM. Envelhecimento populacional: demandas e possibilidades na área de saúde. Demographicas 2016; 153-166.

2. Instituto Brasileiro de Geografia e Estatística (IBGE). Tábua Completa de Mortalidade para o Brasil - 2015. Brasília: MS; 2017.

3. Freitas EV, Py L. Tratato de Geriatria e Gerontologia. Rio de Janeiro: Guanabara; 2016.

4. Campos MTFDS, Monteiro JBR, Ornelas APRDC. Factors that affect the aged people food intake and nutrition. Rev Nutr 2000; 13:157-165.

5. Donini LM, Poggiogalle E, Piredda M, Pinto A, Barbagallo M, Cucinotta D, Sergi G. Anorexia and Eating Patterns in the Elderly. PLoS One 2013; 8(5):e63539.

6. Malafarina V, Uriz-Otano F, Gil-Guerrero L, Iniesta R. The anorexia of ageing: Physiopathology, prevalence, associated comorbidity and mortality. A systematic review. Maturitas 2013; (74):293-302.

7. Instituto Brasileiro de Geografia e Estatística (IBGE). Coordenação de Trabalho e Rendimento. Pesquisa de Orçamentos Familiares: 2008-2009. Análise do Consumo Alimentar Pessoal no Brasil. Brasília: Biblioteca do Ministerio do Planejamento, Orçamento e Gestão, 2011.

8. Kris-Etherton PM, Grieger JA, Etherton TD. Dietary reference intakes for DHA and EPA. Prostaglandins Leukot Essent Fat Acids 2009; 81:99-104.

9. Aune DA, Keum N, Giovannucci E, Fadnes LT, Boffetta P, Greenwood DC, Tonstad S, Vatten LJ, Riboli E, Norat T. Whole grain consumption and risk of cardiovascular disease, cancer, and all cause and cause specific mortality: Systematic review and dose-response meta-analysis of prospective studies. BMJ 2016; 353:i2716.

10. Bernaud FSR, Rodrigues TC. Fibra alimentar: ingestão adequada e efeitos sobre a saúde do metabolismo. Arq Bras Endocrinol Metabol 2013; 57(6):397-405.

11. Huang $\mathrm{T}, \mathrm{Xu}$ M, Lee A, Cho S, Qi L. Consumption of whole grains and cereal fiber and total and cause-specific mortality: Prospective analysis of 367,442 individuals. BMC Med 2015; 13(1):59.

12. Staller K, Song M, Grodstein F, Whitehead WE, Matthews CA, Kuo B, Chan AT. Increased long-term dietary fiber intake is associated with a decreased risk of fecal incontinence in older women. Gastroenterology 2018; 155(3):661-667.

13. Gavanski DS, Baratto I, Gatti RR. Avaliação do hábito intestinal e ingestão de fibras alimentares em uma população de idosos. Rev Bras Obesi Nutr Emagr 2015; 9(49):3-11

14. Vries J, Miller PE, Verbeke K. Effects of cereal fiber on bowel function: A systematic review of intervention trials. World J Gastroenterol 2015; 21(29):8952-8963.

15. Cuesta-Zuluaga J, Mueller NT, Álvarez-Quintero R, Velásquez-Mejía EP, Sierra JA, Corrales-Agudelo V, Carmona JA, Abad JM, Escobar JS. Higher fecal shortchain fatty acid levels are associated with gut microbiome dysbiosis, obesity, hypertension and cardiometabolic disease risk factors. Nutrients 2019; 11(1):51.

16. Hamer HM, Jonkers D, Venema K, Vanhoutvin S, Troost FJ, Brummer R-J. Review article: The role of butyrate on colonic function. Aliment Pharmacol Ther 2008; 27(2):104-119.
17. Dai Z, Zhang Y, Lu N, Felson DT, Kiel DP, Sahni S. Association between dietary fiber intake and bone loss in the Framingham Offspring Study. J Bone Miner Res 2018; 33(2):241-249.

18. Tucker LA. Dietary fiber and telomere length in 5674 U.S. adults: An NHANES study of biological aging. Nutrients 2018; 10(4):400.

19. Mcgill CR, Li VLF, Devareddy L. Ten-year trends in fiber and whole grain intakes and food sources for the United States Population: National Health and Nutrition Examination Survey 2001-2010. Nutrients 2001; 7(2):1119-1130.

20. Stephen AM, Champ MM-J, Cloran SJ, Fleith M, van Lieshout L, Mejborn H, Burley VJ. Dietary fibre in Europe: current state of knowledge on definitions, sources, recommendations, intakes and relationships to health. Nutr Res Rev 2017; 30(2):149-190.

21. Assumpção D, Domene SMÁ, Fisberg RM, Barros MBDA. Qualidade da dieta e fatores associados entre idosos: Estudo de base populacional em Campinas, São Paulo, Brasil. Cad Saude Publica 2014; 30(8):1680-1694.

22. Silva GM, Durante EB, Assumpção D, Barros MBA, Corona LP. Elevada prevalência de inadequação do consumo de fibras alimentares em idosos e fatores associados: um estudo de base populacional. Rev Bras Epidemiol 2019; 22:e190044.

23. Levy-Costa RB, Sichieri R, Pontes NS, Monteiro CA. Disponibilidade domiciliar de alimentos no Brasil: Distribuição e evolução (1974-2003). Rev Saude Publica 2005; 39(4):1-10.

24. Levy-Costa RB, Claro RM, Mondini L, Sichieri R, Monteiro CA. Distribuição regional e socioeconômica da disponibilidade domiciliar de alimentos no Brasil em 2008-2009. Rev Saude Publica 2012; 46(1):6-15.

25. Malta DC, Andrade SSCA, Stopa SR, Pereira CA, Szwarcwald CL, Silva Júnior JB, Reis AAC. Estilos de vida da população brasileira: resultados da Pesquisa Nacional de Saúde, 2013. Epidemiol Serv Saude 2015; 24(2):217-226.

26. Alves MCGP. ISACAMP-2014/2015 [Internet]. 2015 [acessado 2018 nov 10]. Disponível em: https://www. fcm.unicamp.br/fcm/sites/default/files/2018/page/ plano_de_amostragem_isacamp_2014.15.pdf.

27. Fisberg RM, Marchioni DML, Almada CAC. Avaliação do consumo alimentar e da ingestão de nutrientes na prática clínica. Arq Bras Endocrinol Metab 2009; 53(5):617-624.

28. Moshfegh AJ, Rhodes DG, Baer DJ, Murayi T, Clemens JC, Rumpler WV, Paul DR, Sebastian RS, Kuczynski KJ, Ingwersen LA, Staples RC, Cleveland LE. The US Department of Agriculture Automated Multiple-Pass Method reduces bias in the collection of energy intakes. Am J Clin Nutr 2008; 88(2):324-332.

29. Brasil. Ministério da Saúde (MS). Instituto Nacional de Alimentação e Nutrição. Secretaria de Programas Especiais. Registro fotográfico para inquéritos dietéticos - utensílios e porções. Goiânia: UNICAMP; 1996.

30. Carrapato P, Correia P, Garcia B. Determinante da saúde no Brasil: A procura da equidade na saúde. Saude Soc 2017; 26(3):676-689. 
31. Geib LTC. Determinantes sociais da saúde do idoso. Cien Saude Colet 2012; 17(1):123-133.

32. Souza JD, Martins MV, Franco FS, Martinho KO Tinôco AL. Dietary patterns of the elderly: characteristics and association with socioeconomic aspects. Rev Bras Geriatr Gerontol 2016; 19(6):970-977.

33. Silveira EA, Martins BB, Abreu LRS, Cardoso CK. Baixo consumo de frutas, verduras e legumes: Fatores associados em idosos em capital no Centro-Oeste do Brasil. Cien Saude Colet 2015; 20(12):3689-3699.

34. Sistema IBGE de Recuperação Automática (SIDRA) [Internet]. [acessado 2019 set 5]. Disponível em: https://sidra.ibge.gov.br/pesquisa/pnad.

35. Matsudo S, Araújo T, Matsudo V, Andrade D, Andrade E, Oliveira LC, Braggion G. Questinário internacional de atividade f1sica (IPAQ): estudo de validade e reprodutibilidade no Brasil. Rev Bras Ativ Fis Saude 2001; 6(2):5-18.

36. Medeiros F, Xavier A. Inclusão digital e capacidade funcional de idosos residentes em Florianópolis, Santa Catarina, Brasil (EpiFloripa 2009-2010). Rev Bras Epidemiol 2012; 15(1):106-122.

37. Xavier AJ, d'Orsi E, Wardle J, Demakakos P, Smith SG, von Wagner C. Internet Use and cancer-preventive behaviors in older adults: Findings from a Longitudinal Cohort Study. Cancer Epidemiol Biomarkers Prev 2013; 22(11):2066-2074.

38. Confortin SC, Schneider IJC, Antes DL, Cembranel F, Ono LM, Marques LP, Borges LJ, Krug RR, d'Orsi E. Condições de vida e saúde de idosos: resultados do estudo de coorte EpiFloripa Idoso. Epidemiologia e Serviços de saude. Rev SUS Bras 2017; 26(2):305-317

39. King DE, Mainous AG III, Lambourne CA. Trends in dietary fiber intake in the United States, 1999-2008. J Acad Nutr Diet 2012; 112(5):642-648.

40. Thompson FE, Midthune D, Subar AF, McNeel T, Berrigan D, Kipnis V. Dietary intake estimates in the National Health Interview Survey, 2000: methodology, results, and interpretation. J Am Diet Assoc 2005; 105(3):352-363.

41. Papanikolaou Y, Fulgoni VL. Grains contribute shortfall nutrients and nutrient density to older US adults: Data from the national health and nutrition examination survey, 2011-2014. Nutrients 2018; 10(5):534.

42. McAnulty JT, Akabas SR, Thuppal SV, Paxson EE, Saklani S,Tucker, Katherine L, Bailey RL. Fiber Intake varies by poverty-income ratio and race/ethnicity in the US Adults. Nutr Today 2017; 52(2):73-79.

43. Melo NCV, Ferreira MAM, Teixeira KMD. Condições de vida dos idosos no Brasil: uma análise a partir da renda e nível de escolaridade. Oikos 2014; 25(1):4-019.

44. Almeida AN. O acesso aos serviços de saúde pelos idosos no Brasil com base na Pesquisa Nacional por Amostra de Domicílios. J Bras Econ Saude 2015; 7(1):43-52.

45. Damiani TF, Pereira LP, Ferreira MG. Consumo de frutas, legumes e verduras na Região Centro-Oeste do Brasil: prevalência e fatores associados. Cien Saude Colet 2017; 22(2):369-382.

46. Nogueira LR, Morimoto JM, Tanaka JAW, Bazanelli AP. Avaliação qualitativa da alimentação de idosos e suas percepções de hábitos alimentares saudáveis. $J$ Heal Sci 2016; 18(3):163-170.
47. Reedy J, Krebs-Smith SM, Miller PE, Liese AD, Kahle LL, Park Y, Subar AF. Higher diet quality is associated with decreased risk of all-cause, cardiovascular disease, and cancer mortality among older adults. J Nutr 2014; 144(6):881-889.

48. Areosa SVC. Idosos Provedores: a importância dos recursos da aposentadoria para as famílias brasileiras. Barbarói 2015; 45:173-184.

49. Venturini CD, Engroff P, Sgnaolin V, El Kik RM, Morrone FB, Silva Filho IG, De Carli GA. Consumo de nutrientes em idosos residentes em Porto Alegre (RS), Brasil: um estudo de base populacional. Cien Saude Colet 2015; 20(12):3701-3711.

50. Cho SS, Qi L, Fahey Jr GC, Klurfeld DM. Consumption of cereal fiber, mixtures of whole grains and bran, and whole grains and risk reduction in type 2 diabetes, obesity, and cardiovascular disease. Am J Clin Nutr 2013; 98(2):594-619.

51. Cosiales-Buil P, Toledo E, Salas-Salvadó J, Zazpe I, Farràs M, Basterra-Gortari FJ, Diez-Espino J, Estruch R, Corella D, Ros E, Marti A, Gómez-Gracia E, Ortega-Calvo M, Arós F, Moñino M, Serra-Majem L, Pintó X, Lamuela-Raventós RM, Babio N, Gonzalez JI, Fitó M, Martínez-González MA, PREDIMED investigators. Association between dietary fibre intake and fruit, vegetable or whole-grain consumption and the risk of CVD: results from the PREvención con DIeta MEDiterránea (PREDIMED) trial. Br J Nutr 2016; 116(3):534-546.

52. Inzitar M, Doets E, Bartali B, Benetou V, Di Bari M, Visser M, Volpato S, Gambassi G, Topinkova E, De Groot L, Salva A, International Association Of Gerontology And Geriatrics (IAGG) Task Force For Nutrition In The Elderly. Nutrition in the age-related disablement process. J Nutr Heal Aging 2011; 15(8):599-604

53. Gomes AP. Baixa qualidade da dieta de idosos: estudo de base populacional no sul do Brasil. Cien Saude Colet 2016; 21(11):3417-3428.

54. Moreira PRS, Rocha NP, Milagres LC, Novaes JF. Análise crítica da qualidade da dieta da população brasileira segundo o Índice de Alimentação Saudável: uma revisão sistemática. Cien Saude Colet 2015; 20(12):3907-3923

55. Mattos LL, Martins IS. Consumo de fibras alimentares em população adulta. Rev Saude Publica 2000; 34(1):50-55.

56. Souza AM, Pereira RA, Yokoo EM, Levy RB, Sichieri R. Alimentos mais consumidos no Brasil: Inquérito nacional de alimentação 2008-2009. Rev Saude Publica 2013; 47(Supl. 1):190S-199S.

Artigo apresentado em 28/06/2019

Aprovado em 04/12/2019

Versão final apresentada em 06/12/2019

Editores-chefes: Romeu Gomes, Antônio Augusto Moura da Silva 